\title{
Methyl- and methoxysalicylatocopper(II) complexes with 2-aminomethylpyridine
}

\author{
Miroslava Puchoňováa, Milan Mazúr ${ }^{b}$, Dušan Valigura ${ }^{a}$ \\ ${ }^{a}$ Institute of Inorganic Chemistry, Technology and Materials, Faculty of Chemical and Food Technology STU, \\ Radlinského 9, 81237 Bratislava \\ ${ }^{b}$ Institute of Physical Chemistry and Chemical Physics, Faculty of Chemical and Food Technology STU, \\ Radlinského 9, 81237 Bratislava \\ miroslava.puchonova@stuba.sk
}

\begin{abstract}
As a part of our systematic study of copper(II) complexes in presence of biologically active ligands, we concluded to realize experiments with methyl- or methoxysalicylatocopper(II) complexes in presence of 2 -aminomethylpyridine (ampy) ligand. This has resulted in four new complexes of the formula $\mathrm{Cu}(\mathrm{XSal})_{2}(\mathrm{ampy})$ (where XSal are 3-MeSal ${ }^{-}=3$-methylsalicylate, $4-\mathrm{MeSal}^{-}=4$-methylsalicylate, 5 - $\mathrm{MeSal}^{-}=5$-methylsalicylate, or 4-MeOSal ${ }^{-}=4$-methoxylsalicylate anions). The synthesis, elemental analysis and spectral properties (EPR, electronic and infrared) are reported. The $\mathrm{Cu}(\mathrm{II})$ atom in all four complexes is probably coordinated by two nitrogen atom of the bidentate chelating ampy as well as monodentate and/or highly asymmetrically chelating salicylate anions. Based on the analyses it was concluded that the structure of these new compounds could be similar to $\left[\mathrm{Cu}(\mathrm{HCOO})_{2}(\mathrm{ampy})\right]$, or $\left[\mathrm{Cu}\left(\mathrm{CH}_{3} \mathrm{COO}\right)_{2}(\mathrm{ampy})\right]$ that are reported in the literature.
\end{abstract}

Keywords: copper(II) complex, methylsalicylate anion, methoxysalicylate anion, 2-aminomethylpyridine, spectral properties

\section{Introduction}

The study of transition metal systems for the preparation of designed solids has gained momentum over the last few decades due to their potential applications in material science, as well as in medicine (Shukla 2011). The history of copper containing drugs with positive effects to various (e.g. inflammatory, infectious) diseases is colourful and they have been known since ancient times. The modern bioinorganic chemistry of copper is nowadays spread out throughout a wide range of topics but the preparation of new copper complexes and study of their potential biological properties for medicine is one of the most frequent. The solid state properties of the prepared compounds are related to structural arrangement of individual molecular species and to their mutual nonbonding interactions that are frequently related to the type and/or position of different substituents of the used ligand molecules and ions. Copper(II) com- plexes of anti-inflammatory drugs have been found to be more potent than the parent ligand (Sorenson 1976). It was found that copper complexes of salicylic acid and 3-methoxysalicylic acid can act as superoxide dismutase mimetics (O'Connor 2012). Salicylic acid and some its derivatives are known as nonsteroidal anti-inflammatory drugs.

The ligand 2-aminomethylpyridine (known also as picolylamine) can act as a chelating as well as a bridging ligand (Shukla 2011). Generally speaking, the copper complexes with 2-aminomethylpyridine (ampy) are known for their mainly chelating manner of coordination and the bridging mode of bonding to central atoms is rather rare. The latter coordination mode was mostly found in a few $\mathrm{Ag}(\mathrm{I})$ complexes (Yilmaz 2010, Sailaja 2000, Swarnabala, 1997, Bowmaker, 2005, Feazell 2005) and in some rhodium complex (Crawford 1994). Moreover, the central atom oxidation state, the type and number of the other donor atoms, as well as their relative positions within the ligand<smiles>[R]c1ccc(C(=O)O)c(O)c1</smiles><smiles>NCc1ccccn1</smiles><smiles>OCc1ccccn1</smiles><smiles></smiles>

Fig. 1. The formulae of salicylic acid derivatives, 2-aminomethylpyridine and 2-hydroxymethylpyridine. 
are the major factors determining the individual complex molecule activity including its activity in biological environment (Ali 2013). In relation to that our interest is targeted to systematic study of the prepared salicylatocopper complexes with different N-donor ligands that could model different biological function including the antioxidant properties. As the extension of our previous study of the salicylatocopper(II) complexes with 2-hydroxymethylpyridine (2-hydroxymethylpyridine known also as 2-pyridylcarbinol and abbreviated here as hmpy) we are in present paper focusing our attention on the investigation of the relation between the preparation, composition and structure of copper(II) complexes with different methyl-, or methoxysalicylic acid derivatives in presence of the ampy ligand and to the further comparison of the complexes containing pyridine derivatives containing the sidearm with $-\mathrm{NH}_{2}$ or $-\mathrm{OH}$ group. We present here four new complexes, of general formula $\left[\mathrm{Cu}(\mathrm{XSal})_{2}(\mathrm{ampy})\right]$ (where XSal = 3-, 4- or 5-methylsalicylate anion or 4-methoxylsalicylate anion) together with their spectral properties and proposed structure.

\section{Experimental}

\section{Synthesis of complexes}

2-Aminomethylpyridine ( $1 \mathrm{mmol}$ or $0.5 \mathrm{mmol}$ ) was added to water solution of copper(II) acetate $(0.5 \mathrm{mmol})$ under stirring. Methyl- or methoxysalicylic acid ( $1 \mathrm{mmol}$ ) was added to reaction mixture together with necessary amount of solvent (ethanol, methanol, acetonitrile or water). The reaction mixture was stirred for a few hours at room temperature. The precipitates were filtered off, washed with small amount of appropriate solvent and dried in air at ambient temperature. The clear mother liquid solutions were left to crystallize at laboratory temperature. The all samples were characterized by all available spectral methods (electronic and infrared spectroscopy, elemental analysis, EPR spectroscopy).

Anal Calc for $\mathrm{Cu}(3-\mathrm{MeSal})_{2}$ (ampy): C, 55.8; H, 4.7; N,5.9. Found: C, 55.4; H, 4.7; N, 6.2.

Anal Calc for $\mathrm{Cu}(4-\mathrm{MeSal})_{2}(\mathrm{ampy}): \mathrm{C}, 55.8 ; \mathrm{H}, 4.7$; N,5.9. Found: C, 56.7; H, 4.7; N, 4.8.
Anal Calc for $\mathrm{Cu}(5-\mathrm{MeSal})_{2}$ (ampy): C, 55.8; H, 4.7; N,5.9. Found: C, 55.8; H, 4.6; N, 5.7. Anal Calc for $\mathrm{Cu}(4-\mathrm{MeOSal})_{2}($ ampy): C, 52.2; H, 4.4; N,5.5. Found: C, 52.8; H, 4.4; N, 5.0.

\section{Apparatus and equipment}

Analytical grade (Aldrich, or Sigma) chemicals were used without further purification. Carbon, hydrogen and nitrogen analyses were carried out on a CHNSO FlashEA ${ }^{\mathrm{TM}} 1112$ Automatic Elemental Analyzer.

The infrared spectra $\left(4000-400 \mathrm{~cm}^{-1}\right)$ were measured with a NICOLET 5700 FT-IR (Nicolet) spectrophotometer at room temperature using ATR technique.

The electronic spectra $(190-1100 \mathrm{~nm})$ of the complexes were measured in nujol suspension with a SPECORD 250 Plus (Carl Zeiss Jena) spectrophotometer at room temperature.

EPR spectra of the polycrystalline samples were measured with a X-band EPR spectrometer (EMX series, Bruker) at room temperature.

\section{Results and discussion}

The four blue products of unexpected composition were obtained despite the modification of reaction conditions e.g. variation of $\mathrm{Cu}$ : ampy molar ratio and/or changing the used solvent in a contrary to the results found for acetate and formate products (Barquín 2009) where up to the three different molar ratios $\mathrm{Cu}$ : ampy were observed. All complexes under study were characterized by the infrared and electronic spectra. The obtained spectral data show that the composition of the prepared complexes is not affected by changing the reaction conditions. The carboxylate stretching frequencies $\nu_{\mathrm{as}}\left(\mathrm{COO}^{-}\right)$ and $\nu_{\mathrm{s}}\left(\mathrm{COO}^{-}\right)$for all complexes under study are at about 1625 and $1435 \mathrm{~cm}^{-1}$, respectively. Individual data are given in Table 1. Differences between antisymmetric and symmetric stretches $\left(\Delta \nu=\nu_{\mathrm{as}}-\nu_{\mathrm{s}}\right)$ are for all prepared complexes greater than $\Delta \nu$ for the ionic form of the appropriate acid, which is consistent with the monodentate and/or highly asymmetric bidentate bonding mode of carboxylate group (Nakamoto 2009). Moreover, with all of products the absorption bands are rather broad and

Tab. 1. Infrared spectra data $\left(\mathrm{cm}^{-1}\right)$ and solid-state electronic spectra $(\mathrm{nm})$.

\begin{tabular}{|c|c|c|c|c|}
\hline Compound & $v_{\mathrm{as}}\left(\mathrm{COO}^{-}\right)$ & $v_{s}\left(\mathrm{COO}^{-}\right)$ & $\Delta v\left(\mathrm{COO}^{-}\right)$ & $\lambda / \mathrm{nm}$ \\
\hline $\mathrm{Cu}(3-\mathrm{MeSal})_{2}(\mathrm{ampy})(\mathbf{1})$ & 1622 & 1435 & 187 & 635 \\
\hline $\mathrm{Cu}(4-\mathrm{MeSal})_{2}(\mathrm{ampy})(\mathbf{2})$ & 1633 & 1442 & 191 & 635 \\
\hline $\mathrm{Cu}(5-\mathrm{MeSal})_{2}(\mathrm{ampy})(\mathbf{3})$ & 1628 & 1426 & 202 & - \\
\hline $\mathrm{Cu}(4-\mathrm{MeOSal})_{2}(\mathrm{ampy})(\mathbf{4})$ & 1633 & 1443 & 190 & $630,700_{\mathrm{sh}}$ \\
\hline
\end{tabular}


of poor resolution, which allowed us to think about some possibility to of similar bonding mode of the our salicylate anions in comparison to acetate ones in Cu(acetate) ${ }_{2}$ (ampy) (Barquín 2009). For all complexes there is a broad asymmetric pair of medium intensity bands attributable to $\mathrm{N}-\mathrm{H}$ vibrations $\left(\nu_{\mathrm{as}}(\mathrm{NH})\right.$ and $\nu_{\mathrm{s}}(\mathrm{NH})$ at about 3315 and $3253 \mathrm{~cm}^{-1}$, respectively) and broad envelope attributable to system of intramolecular and intermolecular hydrogen bond vibrations within the region of $3000 \mathrm{~cm}^{-1}-2700 \mathrm{~cm}^{-1}$.

The shades of blue colour for complexes (1)-(4) are in good agreement with the electronic spectra that are represented by broad asymmetric complex but unresolved bands with a maximum at about $630 \mathrm{~nm}$. The complexity of the electronic spectra is another result that points out that the central atoms coordination polyhedra in all products probably are less symmetrical than one can expect for the given number of donor atoms. There are also intraligand charge transfer bands $(215-300 \mathrm{~nm})$ and LMCT band in the range of 300-350 $\mathrm{nm}$.
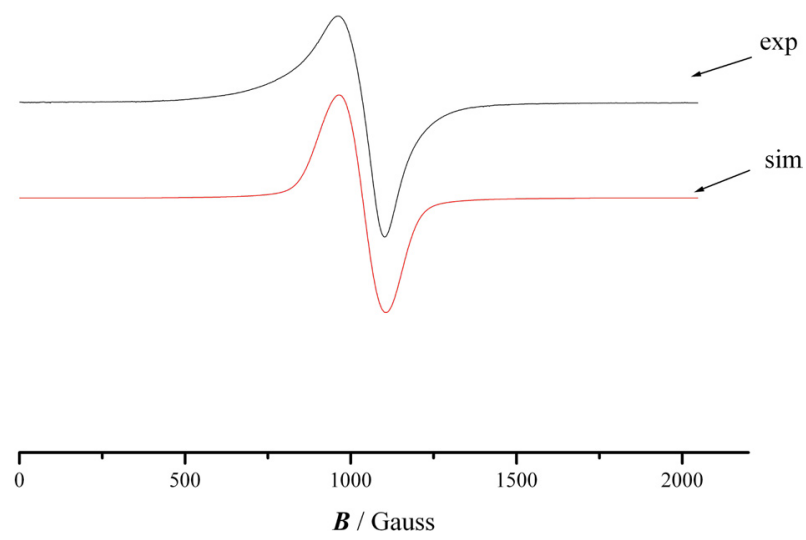

a)
The EPR spectra (Figs. 2 and 3) of powder samples were measured at room temperature. The spectra of mentioned samples are very similar to each other with partly resolved axial symmetry features. The simulation of spectra gave us the $\mathrm{g}$ - factor values (Table 2) in the range of 2.07-2.10 for $g_{\perp}$ and 2.11-2.24 for $g_{||}$, respectively.

The "pseudoaxial" character with $g_{\perp}$ and $g_{||}$values close each to other resulted in $G$ parameters within the narrow range from 1,15 to 2,67 (see Tab 2) and indicate the presence of some exchange coupling between copper(II) atoms having the coordination spheres of distorted tetragonal symmetry that result in $d_{\mathrm{x}^{2}-\mathrm{y}^{2}}$ ground states (Hathway 1970).

Tab. 2. EPR spectra parameter values.

\begin{tabular}{lccc}
\hline Compound & $g_{\|}$ & $g_{\perp}$ & $G$ \\
\hline $\mathrm{Cu}(3-\mathrm{MeSal})_{2}(\mathrm{ampy})(\mathbf{1})$ & 2.115 & 2.100 & 1.15 \\
$\mathrm{Cu}(4-\mathrm{MeSal})_{2}(\mathrm{ampy})(\mathbf{2})$ & 2.181 & 2.071 & 2.55 \\
$\mathrm{Cu}(5-\mathrm{MeSal})_{2}(\mathrm{ampy})(\mathbf{3})$ & 2.248 & 2.093 & 2.67 \\
$\mathrm{Cu}(4-\mathrm{MeOSal})_{2}(\mathrm{ampy})(\mathbf{4})$ & 2.196 & 2.096 & 2.58 \\
\hline
\end{tabular}
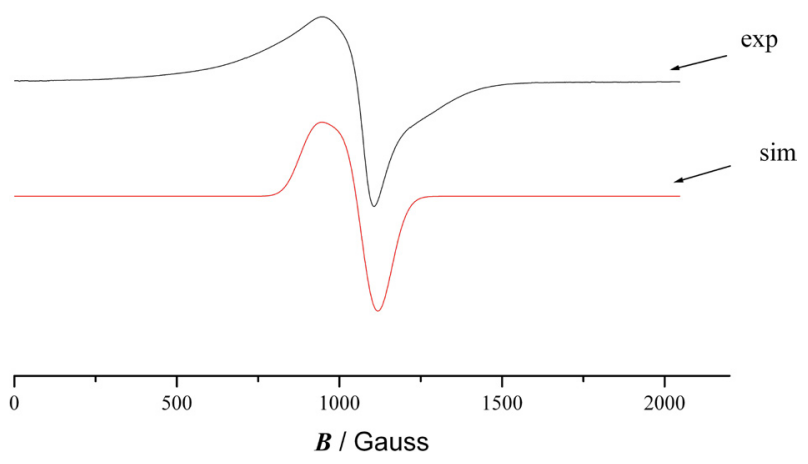

b)

Fig. 2. EPR spectrum a) of $\mathrm{Cu}(3-\mathrm{MeSal})_{2}$ (ampy) (1) b) $\mathrm{Cu}(4-\mathrm{MeSal})_{2}$ (ampy) (2).

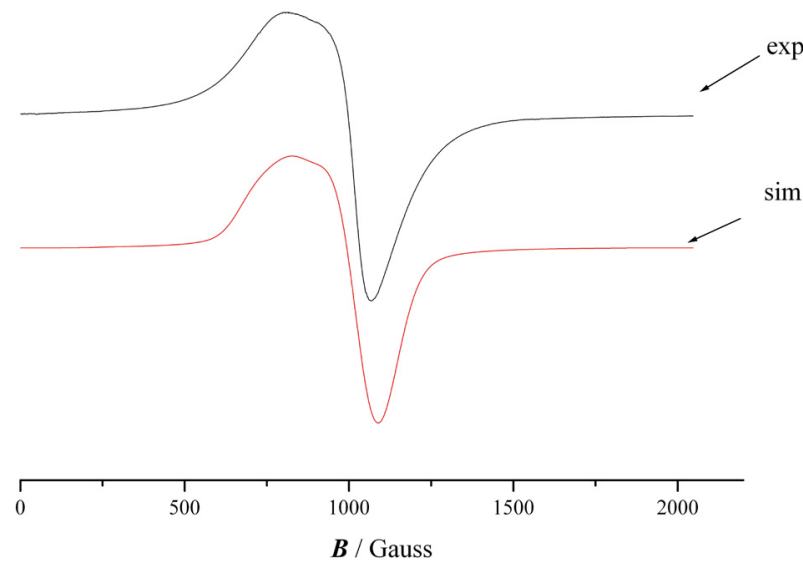

a)
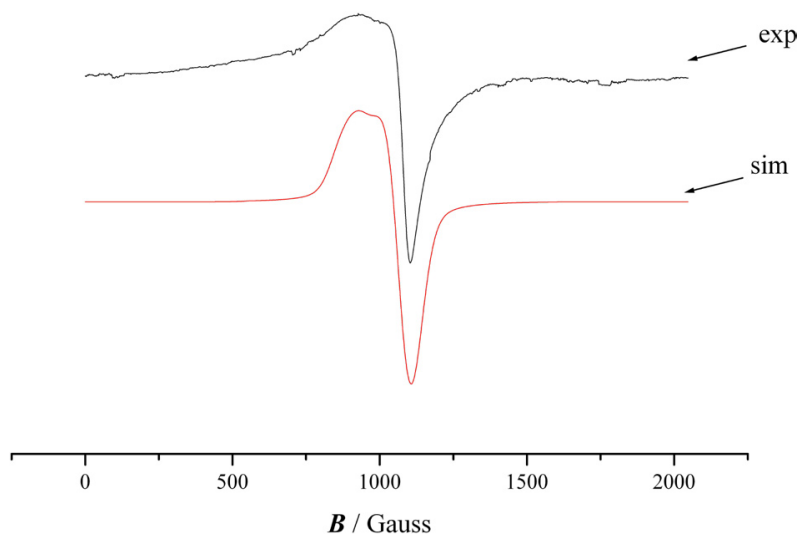

b)

Fig. 3. EPR spectrum a) of $\mathrm{Cu}(5-\mathrm{MeSal})_{2}\left(\right.$ ampy) (3) b) $\mathrm{Cu}(4-\mathrm{MeOSal})_{2}$ (ampy) (4). 
Finally, the obtained data allow us to suggest that the stoichiometrically unusual complexes of composition $\mathrm{Cu}(\mathrm{XSal})_{2}$ (ampy) probably contain the ampy chelating ligand together with the asymmetrically bonded salicylato anions thus giving $\left\{\mathrm{CuN}_{2} \mathrm{O}_{2} \mathrm{O}^{\prime} \mathrm{O}\right.$ " $\}$ chromophores, but that could be proved by solving the structure of individual obtained products, which was up to now unsuccessful. Searching through the Cambridge Crystallographic Database gives some hints. In about a half of all structures (38) of copper(II) complexes containing the ampy ligand moiety the $\mathrm{Cu}$ : ampy molar ratio is $1: 2$, and there are two "extreme" ratios - one example of $1: 3$ ratio and the other one exhibits $2: 1$ stoichiometry. Remain- ing 16 examples are of $1: 1$ stoichiometry, but only 9 of them are truly $1: 1$ and the rest of them are of $1: 1$ as a consequence of some other reasons, e.g. presence of chelating multidentate ligand, or presence of bridging anionic ligand(s). As it was mentioned above in two compounds there are carboxylato anions present together with the ampy ligand $-\mathrm{Cu}(\mathrm{RCOO})_{2}$ (ampy), where $\mathrm{R}=\mathrm{H}$, or $\mathrm{CH}_{3}$ (Barquín 2009). Despite of similarity of the acetate and formate anions the structures are different (Fig 4 and 5).

While formate complex exhibit chain structure (Fig. 4), the acetate one is of molecular structure (distance between the $\mathrm{O} 2$ atom and the neighbouring Cul is close to $3 \AA$ ).

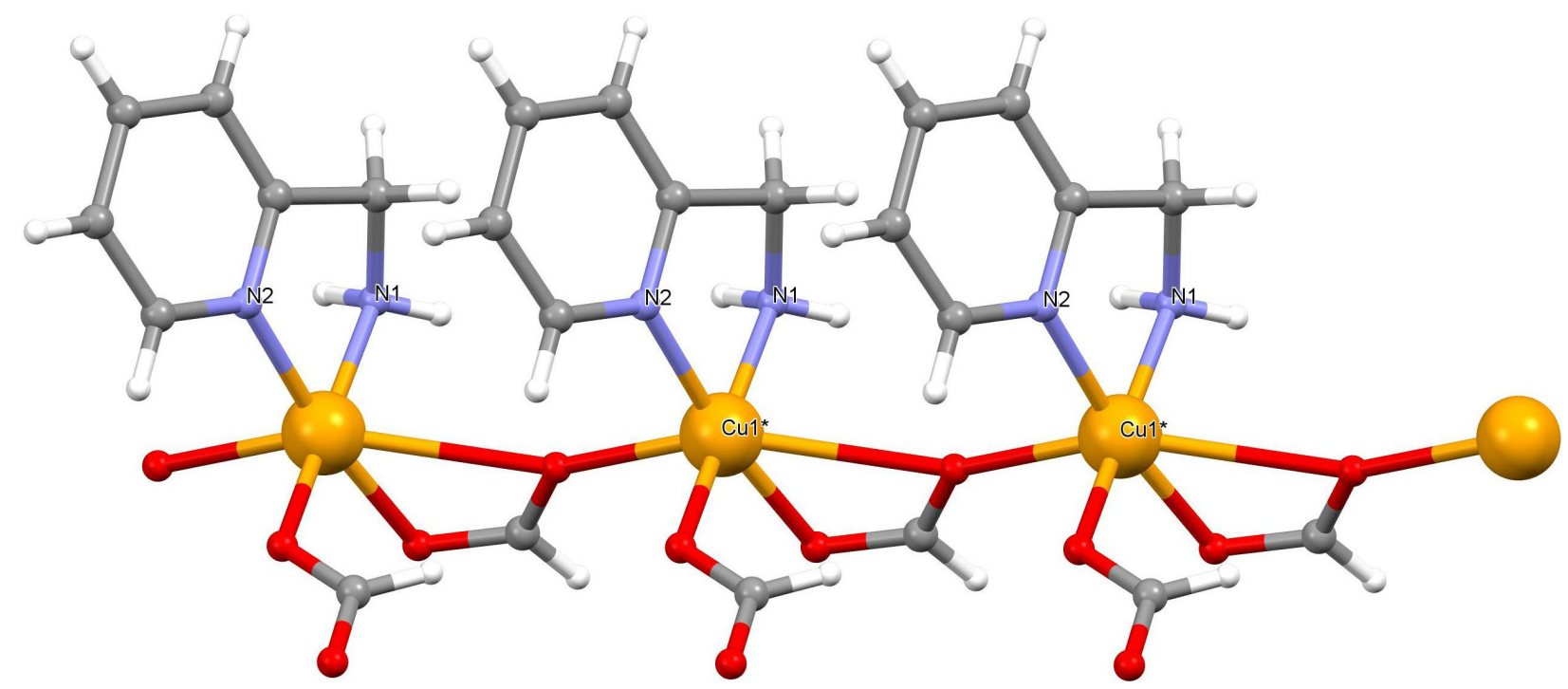

Fig. 4. Crystal structure of $\mathrm{Cu}(\mathrm{HCOO})_{2}$ (ampy) (Barquín 2009).

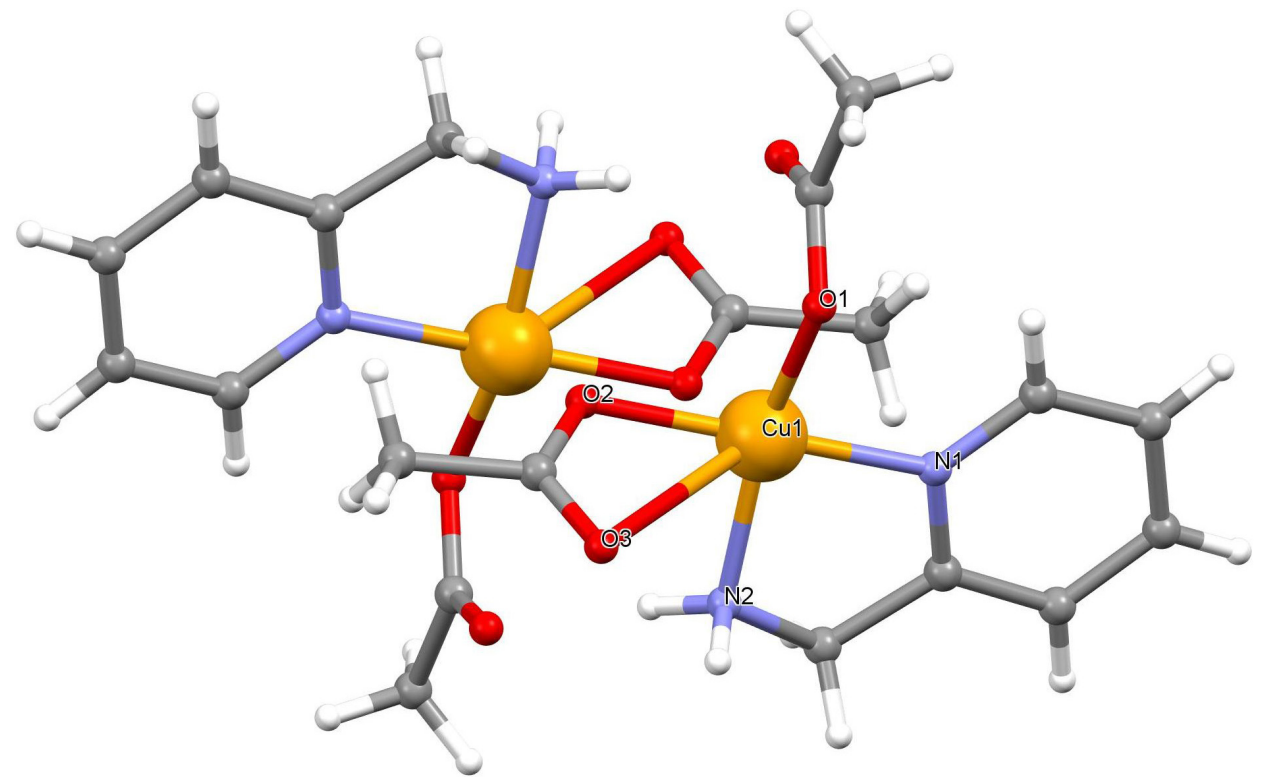

Fig. 5. Crystal structure of $\mathrm{Cu}\left(\mathrm{CH}_{3} \mathrm{COO}\right)_{2}($ ampy) (Barquín 2009). 
Both structures have in common different bonding mode of both anions, asymmetry of the bonding modes and moreover the formation of strong hydrogen bonds between the oxygen atom of the monodentately bonded carboxylate anion with hydrogen atom of the $-\mathrm{NH}_{2}$ group of the neigbouring chain/molecule.

On other hand, only one of the 26 copper(II) complexes with 2-hydoxymethylpyridine (abbreviated as hmpy) that exhibits $\mathrm{Cu}:$ hmpy ratio $1: 1-$ catena- $\left[\mathrm{Cu}\left(\mu_{2}-\mathrm{Cl}\right)-\left(\mu_{2}-\mathrm{hmpy}\right) \mathrm{Cl}\right]_{\mathrm{n}}$. (Lah 2006) All other complexes including carboxylato complexes exhibit $\mathrm{Cu}:$ hmpy ratio $1: 2$. As an example we could point out recently published methyl- and methoxysalicylatocopper complexes with 2-hydroxymethylpyridine of the formula $\left[\mathrm{Cu}(\mathrm{XSal})_{2}(\mathrm{hmpy})_{2}\right]$ where XSal $=3$-methylsalicylate, 4-methylsalicylate or 5-methylsalicylate anion or 3-methoxylsalicylate or 4-methoxylsalicylate anion) (Repická 2012).

In conclusion we can say that despite changing the reaction conditions we have obtained four new complexes of the same stoichiometric formula $\mathrm{Cu}(\mathrm{XSal})_{2}(\mathrm{ampy})$. Based on elemental analyses, spectral data we can conclude that in solid state their structures could be similar to those ones found for $\left[\mathrm{Cu}(\mathrm{HCOO})_{2}(\right.$ ampy $\left.)\right]$, or $\left[\mathrm{Cu}\left(\mathrm{CH}_{3} \mathrm{COO}\right)_{2}(\right.$ ampy $\left.)\right]$.

\section{Acknowledgement}

This work was supported by courtesy of the Slovak Grant Agencies VEGA 1/0388/14 and APVV-0014-11.

\section{References}

Ali HA, Darawsheh MD, Rappocciolo E (2013) Polyhedron 61: 235-241.

Barquin M, Garmendia MJG, Larrinaga L, Pinilla E, Torres MR (2009) Inorg. Chim. Acta 362: 23342340.

Bowmaker GA, Effendy, Nitiatmodjo M, Skelton BW, White AH (2005) Inorg. Chim. Acta 358: 43274341.

Crawford CA, Day EF, Streib WE, Huffman JC, Christou G (1994) Polyhedron 13: 2933-2942.

Feazell RP, Carson CE, Klausmeyer KK (2005) Eur. J. Inorg. Chem. 16: 3287-3297.

Hathaway BJ, Tomlinson AAG, (1970) Coord. Chem. Rev. 5: 1-43.

Lah N, Leban I, Clerac R (2006) Eur. J. Inorg. Chem. 23: 4888-4894.

Nakamoto K (2009) Infrared and Raman spectra of inorganic and coordination compounds, Part B, Sixth Ed. Wiley 118-120.

O’Connor M, Kellett A, McCann M, Rosair G, McNamara M, Howe O, Creaven B S, McClean S, Foltyn-Arfa Kia A, O'Shea D, Devereux M (2012) J. Med. Chem. 55: 1957-1968.

Repická Z, Puchoňová M, Husáriková L, Moncol J, Koman M, Mazúr M, Valigura D (2012) Cent. Eur. J. Chem. 10(5): 1506-1515.

Sailaja S, Rajasekharan MV (2000) Inorg. Chem. 39: 4586-4590.

Shukla M, Srivastava N, Saha S, Rao TR, Sunkari S (2011) Polyhedron 30: 754-763.

Sorenson JRJ (1976) J. Med. Chem. 19: 135-148.

Swarnabala G, Rajasekharan MV(1996) Polyhedron 16: 921-925.

Yilmaz F, Yilmaz VT, Soyer E, Buyukgungor O (2010) Inorg. Chim. Acta 363: 3165-3171. 